Edición Extra-Ordinaria. ISSN 2027-1034 P. p395-403

Memorias del VII Encuentro Nacional de Experiencias en la Enseñanza de la Biología y la Educación Ambiental y II Congreso Nacional de Investigación en la Enseñanza de la Biología

\title{
LA MODELIZACIÓN COMO ESTRATEGIA DIDÁCTICA PARA EL RECONOCIMIENTO DEL PATRIMONIO CULTURAL Y NATURAL DEL MUNICIPIO DE FLORESTA (BOYACÁ) EN LOS ESTUDIANTES DEL COLEGIO TÉCNICO HÉCTOR JULIO RANGEL QUINTERO
}

\section{MODELLING AS A TEACHING STRATEGY FOR RECOGNITION OF CULTURAL AND NATURAL HERITAGE TOWNSHIP FLORESTA (BOYACÁ) IN TECHNICAL SCHOOL STUDENTS HECTOR JULIO RANGEL QUINTERO}

\section{Gutiérrez Rodríguez Laura Melissa ${ }^{1}$}

\section{Escobar Gloria ${ }^{2}$}

\section{Resumen}

Presentamos la propuesta de la práctica pedagógica desarrollada en el Colegio Héctor Julio Rangel Quintero del Municipio de Floresta (Boyacá) con una duración de seis meses y bajo los lineamientos del Grupo de Investigación Conocimiento Profesional del Profesor de Ciencias de la Universidad Pedagógica Nacional (Bogotá-Colombia). La investigación se centró en las estrategias que utilizan los maestros en la modelización para la enseñanza de la Biología en los espacios académicos, permitiendo de a los docentes y estudiantes reflexionar sobre su labor y así mejorar las falencias y fortalecer sus cualidades, y de esta forma "propiciar la meta-reflexión de los profesores acerca de su conocimiento docente con el fin de fortalecer su identidad profesional, (Valbuena, 2009: 3), para ello el proceso investigativo se constituyó a partir de la perspectiva cualitativa, con un enfoque interpretativo donde se empleó diferentes instrumentos de recolección de información.

Finalmente se muestra las conclusiones más relevantes arrojadas por la investigación de la práctica pedagógica.

Palabras Clave: Conocimiento Profesional del Profesor, Modelización, Estrategia Didáctica, Profesor de Biología

\footnotetext{
${ }^{1}$ Estudiante IX semestre de Licenciatura en Biología. Universidad Pedagógica NacionalEstudiante de Licenciatura en Biología) dbi.Igutierrez@pedagogica.edu.co

${ }^{2}$ Profesora del Departamento de Biología. Universidad Pedagógica Profesora de Licenciatura en Biología) araneida542@yahoo.com.mx
} 
Bio-grafía Escritos sobre LaBiología y su Enseñanza.

Edición Extra-Ordinaria. ISSN 2027-1034 P. p395 - 403

Memorias del VII Encuentro Nacional de Experiencias en la Enseñanza de la Biología y la Educación Ambiental y II Congreso Nacional de Investigación en la Enseñanza de la Biología

\section{Abstrac}

We present the proposal of pedagogical practice developed in the College Hector Julio Rangel Quintero Township Floresta (Boyacá) with a duration of six months and under the guidelines of Professional Knowledge Research Group Professor of Sciences National Pedagogic University (Bogotá-Colombia). The research focused on strategies that teachers use in modeling for teaching biology in academic, allowing teachers and students to reflect on their work and improve the weaknesses and strengthen their qualities, and thus "promote the meta-reflection of teachers about their teaching knowledge in order to strengthen their professional identity, (Valbuena, 2009: 3), for which the research process was established from the qualitative perspective, with an interpretive approach which used different data collection instruments.

Finally, we show the most relevant conclusions from the research dumped pedagogical practice.

Key word: Teacher Professional Knowledge, Modeling, Didactic Strategies, Biology Teacher.

\section{Introducción}

La Universidad Pedagógica Nacional, educadora de educadores, es una entidad de carácter público, que desde los diferentes marcos institucionales se ha consolidado como líder en la formación de maestros de las diferentes ciencias, contribuyendo a la investigación, producción y divulgación del pensamiento pedagógico y didáctico. Dentro este panorama el proyecto curricular de la Licenciatura en Biología se concibe como un proyecto de investigación educativa que le ofrece a los futuros maestros ambientes de formación que abarca el campo disciplinar, humanístico y pedagógico en cada uno de sus semestres los cuales son orientados desde diversos espacios, para así poder formar maestros que se puedan desenvolver en múltiples situaciones problema. Además promueve el fortalecimiento de la investigación pedagógica los cuales contribuyen a la construcción de un maestro integral.

Para el caso específico del presente trabajo, se realizóbajo los lineamientos del grupo de investigación Conocimiento Profesional del Profesor de Ciencias, bajo el marco de la práctica integral pedagógica en donde se abordaron problemas reales de la educación con el fin de brindar una intencionalidad formativa como futuros maestros. El objetivo general de la investigación es: "Desarrollar una propuesta de enseñanza teniendo en cuenta la modelización empleada por los maestros que permita el reconocimiento del patrimonio cultural y natural por parte de los estudiantes del Colegio Técnico Héctor Julio Rangel Quintero del Municipio de Floresta (Boyacá)" y como pregunta problema:"¿Qué papel desempeña una 
Bio-grafia Escritos sobre laBiología y su Enseñanza.

Edición Extra-Ordinaria. ISSN 2027-1034 P. p395 - 403

Memorias del VII Encuentro Nacional de Experiencias en la Enseñanza de la Biología y la Educación Ambiental y II Congreso Nacional de Investigación en la Enseñanza de la Biología

propuesta de enseñanza teniendo en cuenta el uso de la modelización empleada por los maestros para permitir el reconocimiento del patrimonio cultural y natural por parte de los estudiantes del Colegio Técnico Héctor Julio Rangel Quintero del Municipio de Floresta (Boyacá)?

La investigación, se centra en las estrategias que utilizan los maestros en la modelización para la enseñanza de la Biología en los espacios académicos, permitiendo de a los docentes y estudiantes reflexionar sobre su labor y así mejorar las falencias y fortalecer sus cualidades, y de esta forma "propiciar la meta-reflexión de los profesores acerca de su conocimiento docente con el fin de fortalecer su identidad profesional, (Valbuena, 2009: 3).

\section{Metodología}

Esta investigación se realizó en el primer periodo del 2013 en el Colegio Héctor Julio Rangel Quintero del Municipio de Floresta (Boyacá), enmarcada dentro de la perspectiva cualitativa-interpretativa, donde "la relación entre el investigador y los sujetos investigados toma relevancia, partiendo de la realidad teniendo en cuenta comportamiento, conocimientos, actitudes, etc". (Bonilla y Rodríguez, 1997: 50), además "el investigador reconoce que la despersonalización no es posible, y que, como meta principal es trabajar de manera comprometida con la comunidad, para permitir que aflore y se pueda sistematizar la vivencia y el conocimiento que ellos tienen de su realidad." (Bonilla y Rodríguez, 1997: 52), permitiendo de esta manera un acercamiento a la problemática que se desarrolló en el transcurso de la investigación, pues ella "rescata y asume la importancia de la subjetividad, la intersubjetividad es vehículo por medio del cual se logra el conocimiento de la realidad humana" (Galeano, 2009: 21) y mediante un enfoque interpretativo, pues como Cohen y Manion (1991), nos plantean que este "se realiza a pequeña escala estudiando al individuo, más que estadística es subjetiva, comprende las acciones y los significados".

En cuanto a los procesos metodológicos, se llevaron a cabo por medio de 2 instrumentos principalmente:La observación: debido a que "es un procedimiento fácil de aplicar, directo y que exige técnicas de tabulación muy sencillas" (Cerda, 1993: 237) y siendo ésta "una de las formas más directa e inmediata de conocer los fenómenos y las cosas" (Cerda, 1993: 237), aunque es preciso aclarar, como nos dice Cerda (1993), que como todo proceso riguroso, exige por parte del observador una actitud, una postura y un fin determinado en relación con las cosas que observa. La entrevista fue el segundo instrumento dado que es "una modalidad de interrogación, que se refiere al acto de hacer preguntas a alguien con el propósito de obtener un tipo de información específica", complementando de esta manera la observación puesto que "por medio de ella se obtiene toda aquella información que no obtenemos por la observación, porque a través de ello podemos penetrar en el mundo interior del ser humano y conocer sus 
Bio-grafía Escritos sobre LaBiología y su Enseñanza.

Edición Extra-Ordinaria. ISSN 2027-1034 P. p395 - 403

Memorias del VII Encuentro Nacional de Experiencias en la Enseñanza de la Biología y la Educación Ambiental y II Congreso Nacional de Investigación en la Enseñanza de la Biología

sentimientos, su estado anímico, sus creencias, sus conocimientos, etc." (Cerda, 1993: 258-259).

Los datos y resultados obtenidos por la implementación de los instrumentos se recolectaron apoyándose en un cuaderno de y dispositivos mecánicos que permitieron tener un registro sonoro, fotográfico o fílmico de los diversos aspectos estudiados. (Cerda, 1993: 251)

En la presente investigación se presentaron unas fases en las que se fue indagando y aplicando lo planteado anteriormente, dichas fases son las siguientes:

- Fase 1: Indagación

- Fase 2: Diseño de la propuesta

- Fase 3: Implementación de la Propuesta

\section{Resultados Y Discusión}

De acuerdo a las observaciones realizadas durante la práctica en el Municipio de Floresta (Boyacá) de las diferentes clases de Biología del grado sexto, se logró identificar que las profesoras no tienen claro o no son conscientes de la utilización de la modelización en sus clases. Pues por medio de las entrevistas realizadas se pudo deducir que las respuestas proporcionadas por ellas no coinciden con la realidad, en la medida que lo observado por parte de la investigadora no concuerda con los resultados obtenidos por la aplicación del instrumento.

En lo que respecta a la profesora Número 1, se puede evidenciar que las respuestas proporcionadas en la entrevista realizada, se aleja en gran medida de la realidad, pues como se muestra en la pregunta número dos (2), al momento de preguntarle cuales son las estrategias didácticas utilizadas por parte de esta maestra en su labor, ella responde enumerando gran variedad de éstas, tales como talleres, videos, láminas, salidas de campo y exposiciones, pero al momento de la clase se puede observar que la maestra en su metodología emplea talleres con guías en donde los estudiantes se remiten solo a copiar de un libro a sus cuadernos sin realizar ni un análisis ni reflexión sobre aquello que copian y videos que puedan aclarar dudas existentes del tema visto, permitiéndonos afirmar lo antes dicho. La pregunta ocho (8) también presenta algunas incongruencias con la realidad pues al preguntarle que otros espacios, aparte del salón, menciona tanto lugares internos como externos de la institución, pero en todo momento el aula es el único escenario empleado.

Con respecto a las preguntas 5,6 y 7 que se refieren específicamente al empleo de estas estrategias y el beneficio de la aplicación de ellas, la profesora nos responde que el empleo de ellas permite un mejor dinamismo de las clases, evitando la monotonía y el tedio, pero como se mencionó anteriormente, no es así. 
Bio-grafía Escritos sobre laBiologia y su Enseñanza.

Edición Extra-Ordinaria. ISSN 2027-1034 P. p395 - 403

Memorias del VII Encuentro Nacional de Experiencias en la Enseñanza de la Biología y la Educación Ambiental y II Congreso Nacional de Investigación en la Enseñanza de la Biología

Pues los mismos estudiantes argumentan que la clase de naturales es una rutina, en donde ya se saben cómo inicia y termina la clase.

Esta metodología empleada por la profesora no moviliza mucho el desarrollo adecuado de la clase, en la medida que al presentarse una monotonía en sus clases y el grupo al tener una actitud calmada y pasiva, no promueve el desarrollo en ellos de una actitud propositiva, ni tampoco ayuda mucho en la comprensión en algunos temas que presentan una mayor dificultad al tener un cierto grado de abstracción como por ejemplo La Célula.

Por último, las preguntas que tienen que ver con los temas específicos de evolución y fósiles, que son las 9, 10 y 11, esta maestra dice que lo evade por el simple hecho de no creer en ello, por ir en contra de sus creencias religiosas.

En lo que respecta la profesora 2, se ve que al igual que en la maestra 1 , se presentan ciertas incongruencias pues en la pregunta dos (2) en donde se pregunta que si utiliza estrategias didácticas en su clase, ella responde "si, todas las que convierten la enseñanza en una pedagogía activa", pero si entramos a comparar con lo observado vemos que la maestra utiliza una metodología basada más en un modelo tradicionalista en donde los estudiantes actúan en ocasiones como simples receptores y la maestra como simple emisora de una saber.

La pregunta ocho (8) también presenta algunas distorsiones, pues al preguntarle qué otros espacios utiliza, a parte del salón, nos dice que aunque con restricciones, hace uso de algunos lugares del colegio, pero de acuerdo a lo evidenciado por la investigadora, el aula es el único escenario empleado.

También es claro que ella, en lo que respecta a las preguntas 5,6 y 7 , que se refieren al empleo de esta estrategias y el beneficio de la aplicación de ellas, esta maestra, al igual que la número 1 , nos comenta que las utiliza para hacer más llevaderas las clases, pero como se mencionó anteriormente no es así.

\section{Discusión}

Con la implementación del instrumento (entrevista) se buscó mirar qué entienden por estrategias didácticas las maestras de Biología del Colegio Héctor Julio Rangel Quintero del Municipio de Floresta Boyacá y más si ellas dentro de sus metodologías hacen uso de la modelización como estrategia didáctica en la enseñanza de esta disciplina.

Por medio de las preguntas que fueron diseñadas en la entrevista se buscó mirar los diferentes componentes que hacen parte del Conocimiento Profesional del Profesor de Ciencias y que tan conscientes están ellas de éstos y su importancia en todo lo relacionado con la enseñanza, pues como se sabe el Conocimiento 
Bio-grafía Escritos sobre LaBiología y su Enseñanza.

Edición Extra-Ordinaria. ISSN 2027-1034 P. p395 - 403

Memorias del VII Encuentro Nacional de Experiencias en la Enseñanza de la Biología y la Educación Ambiental y II Congreso Nacional de Investigación en la Enseñanza de la Biología

Profesional del Profesor requiere la interrelación de diferentes tipos de conocimientos para que éste pueda abarcar todos los campos que están involucrados en la educación. Dentro de estos podemos encontrar el conocimiento disciplinar, contextual, pedagógico y didáctico, los cuales son indispensables que sean poseedores por parte del maestro sin importar qué tema vaya a enseñar.

A través de las observaciones de la clase se pudo evidenciar que en cuanto a la metodología empleada por la docente $\mathrm{N}^{\circ} 1$ se encuentra la aplicación de talleres en clase, cuestionarios para la casa y videos que permitan aclarar dudas si existen frente al tema que se esté enseñando. En las clases de Naturales la profesora utiliza muy poco el tablero pues no realiza muchas explicaciones argumentando que los temas que se dan en Biología son fácil y no se necesita de explicaciones sino que con tan solo el desarrollo de talleres y cuestionarios es suficiente para que los estudiantes aprendan sobre el tema. Con respecto a la Química la maestra si hace explicaciones en el tablero dado que para ella en esta materia si se necesita de éstas para ser entendida pues ésta es mucho más difícil de comprender y asimilar debido a que es un poco más abstracta y compleja que la Biología al estudiar fenómenos que no se pueden ver.

Esta maestra no hace uso de estrategias como la modelización en sus clases, sabiendo que como nos dice Harrison y Treagust (1996) ésta es necesaria en la enseñanza y comprensión de la mayoría de los conceptos científicos, fenómenos o eventos de la ciencia, además que puede ser considerada como una especie de modelo metafórico o analógico, debido a que estos tienen en su estructura concreta una o más cualidades análogas a la estructura de lo representado o modelizado.

Esta dinámica empleada por la maestra número 1 no favorece mucho a la movilización de pensamiento en los estudiantes del grado sexto, dado que ellos se caracterizan por ser un grupo calmado, con poca iniciativa en el momento de proponer actividades que ayuden a promover y facilitar los temas que se ven en la asignatura de Naturales, pero dispuestos a la hora de desarrollar las actividades planteadas. Siempre esperan que la maestra sea la persona que disponga de la dinámica de la clase y muy pocas veces ponen objeción frente a lo propuesto. Son receptivos a las propuestas hechas por la maestra de Naturales.

\section{Conclusiones}

Al terminar este trabajo investigativo se puede concluir:

- No se lograr identificar que las profesoras tengan claro el concepto de modelización, pues cuando se les preguntan por algo relacionado con el tema, las maestras no responden acertadamente, o si lo hacen, lo hacen de una manera muy superficial. 
Bio-grafía Escritos sobre LaBiología y su Enseñanza.

Edición Extra-Ordinaria. ISSN 2027-1034 P. p395 - 403

Memorias del VII Encuentro Nacional de Experiencias en la Enseñanza de la Biología y la Educación Ambiental y II Congreso Nacional de Investigación en la Enseñanza de la Biología

- Aunque las maestras siempre dicen que tratan de innovar y cambiar sus dinámicas de clase para no fomentar la monotonía ni el tedio, al momento de realizar la clase siempre utilizan las mismos recursos pedagógicos, aunque justifican que lo hacen porque el colegio no les brinda más ayudas, ellas como maestras deben buscar nuevas formas y se recursivas con lo que se tenga a la mano.

- A pesar que el Municipio cuenta con una gran riqueza fosilífera que hace parte de su patrimonio cultural y natural, las dos maestras no intentan acercar a los niños a él, ni tratan de aprovechar las ventajas que ellos ofrecen para explicar temas relacionados con La teoría Evolutiva, por la razón de que en contra de sus creencias religiosas, negándoles a ellos la posibilidad de conocer y apropiarse de su patrimonio y con ello partencia hacia su municipio.

- En muchas ocasiones los profesores tan solo se quedan en el discurso sobre la importancia de tener e implementar diferentes formas y estrategias didácticas en el momento de la práctica docente, pues al momento del desarrollo de la clase, siguen con lo mismo que se ha visto, con los mismos modelos tradicionalistas en donde no se busca una movilización de pensamiento ni que los chico desarrollen actitudes propositivas.

- La modelización, al ser una forma más plausible de comprender intuitivamente los conceptos, fenómenos, procesos o eventos, ayuda a comprender mejor los temas que se enseñan en la Biología.

\section{Referencias Bibliográficas}

- Alcaldía Municipal de Floresta. 2005. Esquema de Ordenamiento Territorial Municipio de Floresta. Proyecto de Acuerdo Honorable Concejo Municipal de Floresta Departamento de Boyacá.

- Bender, G, DEFAGO, A. \& CUTRERA, G. 2008. Modelos de enseñanza aprendizaje en futuros profesores de biología. III Congreso Internacional De Enseñanza De La Biología. Mar del Plata Buenos Aires Argentina.

- BONILLA, E. \& RODRíGUEZ, P. 1997. La investigación en Ciencias Sociales. Más allá del dilema de los métodos. Ediciones Uniandes. Grupo Editorial Norma.

- CERDA, H. 1993. Los elementos de la investigación: cómo reconocerlos, diseñarlos y construirlos. Editorial El Búho. 
Bio-grafía Escritos sobre LaBiología y su Enseñanza.

Edición Extra-Ordinaria. ISSN 2027-1034 P. p395 - 403

Memorias del VII Encuentro Nacional de Experiencias en la Enseñanza de la Biología y la Educación Ambiental y II Congreso Nacional de Investigación en la Enseñanza de la Biología

- CHAMizO, J. \& GARCíA, A. 2010. Modelo y Modelaje en la Enseñanza de las Ciencias Naturales. Facultad de Química. Universidad Nacional Autónoma de México. México

- Departamento Nacional de Planeación y Federación Colombiana de Municipios. (2006). Información Municipal para la Toma de decisiones. Floresta Boyacá. Censo Municipal. Alcaldía Municipal.

- FLÓREZ G, et. al. 2009. Modelización de procesos de enseñanza en profesores de ciencias de la ciudad de Manizales (Colombia) desde el concepto contenido pedagógico del conocimiento. Universidad de caldas.

- GALEANO, M. 2009. Estrategias de investigación social cualitativas. El giro en la mirada. La Carreta Editores E.U. Medellín Colombia.

- GRANDE E, CHARRIER M \& VILANOVA, S. 2008. Las representaciones de gen, cromosoma y meiosis que presentan los estudiantes universitarios. III Congreso Internacional De Enseñanza De La Biología. Mar del Plata Buenos Aires Argentina.

- LEVIN H. 2006. The Hearth Trough Time. Fifth and Eighth edition. (R. Torres, trad.) Bogotá, Colombia: Universidad Pedagógica Nacional. (2010).

- LOIZAGA N, SOlANGE S \& CELESTE M. 2008. Los modelos didácticos que subyacen en las prácticas de los profesores de las cátedras de química general y química general y biológica (FACENA - UNNE). III Congreso Internacional De Enseñanza De La Biología. Mar del Plata Buenos Aires Argentina.

- MEDINA L. et. al. 2009. Modelos mentales al interrelacionar los conceptos de célula y ecosistema, utilizando a los insectos como eje transversal. Tecné, Episteme y Didaxis: TED No. Extraordinario. $4^{\circ}$ Congreso Internacional sobre Formación de Profesores de Ciencias.

- MOREIRA, M., GRECA, I. \& RODRíGUEZ. 2002. Modelos Mentales Y Modelos Conceptuales En La Enseñanza \& Aprendizaje De Las Ciencias. Revista Brasileira de Pesquisa em Educação em Ciências, Porto Alegre. V 2 (3), p. 37-57.

- RENDÓN, C. et al. 2008. La elaboración de representaciones concretas tridimensionales como recurso didáctico para el aprendizaje de biología 
Bio-grafía Escritos sobre laBiología y su Enseñanza.

Edición Extra-Ordinaria. ISSN 2027-1034 P. p395 - 403

Memorias del VII Encuentro Nacional de Experiencias en la Enseñanza de la Biología y la Educación Ambiental y II Congreso Nacional de Investigación en la Enseñanza de la Biología

molecular y celular de alumnos de escuela media. III Congreso Internacional De Enseñanza De La Biología. Mar del Plata Buenos Aires Argentina.

- ROJAS Y. 2005. Dificultades en la modelización didáctica del modelo biológico de flor. Un estudio de caso en la licenciatura en educación básica, énfasis en ciencias naturales y educación ambiental de la Universidad de Antioquia. Facultad de Educación. Departamento de Educación Avanzada. Universidad de Antioquia.

- SHULMAN, L. 1987. Knowledge and teaching: foundations of the new reform. Harvard EducationalReview, 57(1), 1-22. Traducción castellana (2005): Conocimiento y Enseñanza: Fundamento de la Nueva Reforma. Profesorado. Revista de Currículum y Formación de Profesorado. V 9. (2),

- STENHOUSE, L., 1987. La investigación como base de la DES: Londres. Enseñanza. (Morata S.S.: Madrid).

- VAlBuenA, E. 2007. El Conocimiento Didáctico del Contenido Biológico. Estudio de las concepciones disciplinares y didácticas de futuros docentes de la Universidad Pedagógica Nacional (Colombia). Tesis doctoral. Universidad Complutense de Madrid 